\title{
Global and Local Skew Detection of Handwritten Gurmukhi Script
}

\author{
Mandeep Kaur \\ GTBKPC \\ Muktsar(Punjab)
}

\author{
Lovnish Bansal \\ BMSC \\ Malout (Punjab)
}

\author{
Jagdeep Singh \\ BMSC \\ Muktsar (Punjab)
}

\begin{abstract}
ASTRACT
This paper presents a new and efficient method for detection and removal of Global and Varying (Local) Skew. Varying (Local) Skew comes into existence in handwritten script. The proposed method is based on Hough transform and windowing. The proposed method divides the Skewed document into horizontal windows. Firstly, for each window the values of skew angle is calculated using Hough transform and then average of skew angles is estimated which represents the Skewness of document. Orientation of text is also calculated. Finally, Document is rotated based on average value of skew angle and orientation. The proposed method calculates skew for both printed and handwritten script. The proposed method can also calculate Multiskew. Experiments and results show that proposed method has high accuracy and can work with degraded papers
\end{abstract}

\section{General terms}

Optical character recognition

\section{Keywords}

Skew, Global skew, Local skew, OCR

\section{INTRODUCTION}

In past, Manual system is used to maintain the records and information in offices. Manual system is not efficient way for keeping the records because it has many disadvantages- time consuming process ,low security, low efficiency, inconsistency of data, difficult to recover in case failures, difficult to perform operation like editing, searching etc. Maintenance of manual system is challenging task. That's why, every field is moving from paper work to electronic documents, this process is known as automation of office documentation. Devices such as text readers, scanner and OCR (Optical Character Recognition) systems are used in the automation of office documentation. Scanner are used to convert the image into electronic data and Optical character recognition is a process by which scanned images of printed text, handwritten text, picture and graphs are converted into a computer recognizable form.

In OCR one of the major problem is Skewness. Skew detection and removal task is done at preprocessing stage of optical character recognition because accuracy of the results of subsequent stages of OCR systems depends upon accuracy of pre-processing stages. Skewness has many effects-it makes document analysis, document understanding, even visualization tasks more difficult. It decreases the efficiency and accuracy of optical character recognitions. That's why removal of skewness is very important for accuracy of OCR

Skew in OCR is defined in two ways. It can be the tilt in bitmapped images of scanned images OR it can be defined as the alignment of lines of text with respect to the horizontal. Skew can have either positive value or negative value. Skew is divided into three types
1. Single skew

2. Multiskew

3. Non uniform skew

Single skew is further divided into two parts Global and Local skew. Global skew mostly comes into existence in printed text. But Local(varying)Skew ,Multiskew and Non uniform skews are found in handwritten text. Global Skew detection of printed text is easier than Varying skew and Multiskew. Because all line are parallel to each other and have same value of skew angle. But in handwritten lines are not parallel to each other that's why every line can have its own skew angle and gap between the lines are not constant

\subsection{Exiting Methods}

Skew Detection and correction techniques are based on

I. Hough Transform

II. projection profile

III. clustering of nearest neighbor

In the projection profile technique [9] the document is projected at different angles. Then peaks (due to text line positions) and troughs (due to inter-line gap positions)are identified. The angle which gives the maximum difference between the peaks and troughs is accepted as the skew angle. The method, being computationally expensive, has been improved by Baird [3] by proposing a quick convergence of this iterative approach. In another approach Akiyama and Hagita [2] partitioned the image vertically into a number of strips. The horizontal projection profile is then calculated for each strip where, from the correlation of the profiles of the neighboring strips, the skew angle is estimated. This yields fast but not so accurate result.

Hashizume et al.[5] first proposed a nearest-neighbor based method. The connected components are detected first. The direction vector of all nearest-neighbor pairs of connected components are accumulated in a histogram, and the peak in the histogram gives the dominant skew. This method is generalized by O'Gorman[6], in which the nearest-neighbor clustering is extended to $\mathrm{K}$ neighbors for each connected components. Because of the use of $\mathrm{K}$ neighbors connection that may be made across text lines, the resultant histogram peak may not be very accurate generally. The algorithm proposed by Liolios et al. [7] attempted to group all components that belong to the same text line into one cluster. Because the average height and width of the components are applied in the process, the method can only cope with documents with a rather uniform font size.

Srihari S. N. and Govindraju V[1] has proposed skew detection method based on the Hough Transform (HT). The HT is computed at all angles of theta between 0 and 180 degrees. A heuristic measures the rate of change in accumulator values at each value of theta. The skew angle is set to the value of that maximizes the heuristic. These methods are computationally very expensive. In [4] only 
bottom pixels of candidate objects within a selected region are fed to Hough transforms. Chaudhary and Pal [8] have proposed a technique for Indian language scripts in which exploits the inherent properties of the script to determine the skew angle. The idea is to detect skew angles of these head lines of scripts. The method detecting skew angles in range ($45^{\circ}$ to $45^{\circ}$ ) Manjunath et al. [11] also used Hough transform to detect the skew angle in two steps. Initially, they identified character blocks from document images and thinning process is performed over all regions. Then next thinned conditions are fed to Hough transform. The primary disadvantage of this technique is that time complexity does not include the thinning process time.Loveleen [9] proposed a method which is based on the image top. The technique is developed by using the concept of Hough Transform. The method deals with an accurate measure of skew, within-line, and betweenline spacing and locates text lines and text blocks. The proposed method is fast as compared to other HT based methods. The noises and the deviation in the document resolution or types are still the main two challenges.

There are many other methods. Omar [10] proposed skew detection and correction technique for Arabic document images based on centre of gravity. It involved inscribing the text in the document by an arbitrary polygon and derivation of the baseline from polygon's centroid. Das et. al, [12] proposed a method based on morphology in which they used run-length smoothening (RSLA) algorithm that is, a closing of the

image using line structuring element that forms solid black bands corresponding to text lines. They opened and closed image to remove the bumps and registered all transitions which provide lines. The skew is computed for the biggest line. This method works better on small skew angles but the time complexity of the method is large. Chen et. al,[13] proposed the method that starts with threshold reduction then by applying recursive morphological closing and openings to close up the text lines. Then remove ascender and descenders, and determine the connected components. Fit a best line for the points in each set of connected components. Estimate the global skew based on the average of the lines after discarding the outlier lines. They reported the skew error to be larger than $0.3^{\circ}$

\section{PROPOSED METHOD}

This section presents the proposed methodology for detecting global and local skew of gurmukhiscript. Methodology is based on Hough transform, coordinate geometry and Windowing. It deals with both global skew as well as local. It has been seen detection of global skew is easier. But Local skew is still challenging tasks because handwritten text lines are not parallel to each other.

The algorithm based on Hough transform and windowing. It firstly removes noise from scanned image. In next step it divides the image into small horizontal windows. Windowing is performed because in local skew every line can have its own skew angle. To calculate the skew angle of every line, page is divided into small segments which are called windows. Window basically contains text line. The algorithm is divided into two sub parts-Skew Angle detection and Orientation detection. Skew angle algorithm calculates skew angle for every window and Orientation algorithm calculates orientation of text.

Skew Angle Algorithm: Skew Angle algorithm is used to find the magnitude of skew angle made by text line with horizontal. For each window, it runs Hough transform to find the longest straight line in the window .After finding the longest line, Calculate distance of start and end point of line from origin. Skew angle is calculated using following formula

$$
\begin{gathered}
\mathrm{m}=\text { DeltaX/ DeltaY } \\
\theta=\operatorname{atand}(\mathrm{m})+2 * \mathrm{~m}
\end{gathered}
$$

Orientation Algorithm: It gives direction of text lines whether text lines are going upward or downward. The direction of line tells about the type of skewness. If lines are going upward direction it is positive skew otherwise it is negative skew. It runs the Hough transform on whole document to find the longest straight line. After comparing the start and end point of straight line, it gives the orientation value.

\section{Algoritm1:Skew detection based on HT and horizontal windowing}

Step1: Read the scanned image and convert it into Binary Image.

Step2: Crop the image to find the area of interest.

Step3: Remove noise from image using motion filter.

Step4: Divide the image into small horizontal windows.

Step5: For each window run Skew Angle algorithm to get the value of skew angle.Calculate average value of skew angle as given below

Skew Angles =sum of skew angles/number of windows Step6: To find skew orientation of page run Orientation algorithm

Step7: Correct the skew by rotating the image according

to average value of skew and orientation.

\section{EXPERIMENTAL RESULTS}

This section presents the results of the experiments conducted to study the performance of the proposed method. I have taken different samples of skewed documents from different sources like handwritten notes, textbooks, newspapers and the like. Obtained Skew Angle by the proposed methodology for these documents are reported in Table 1. Following are samples of corrected images

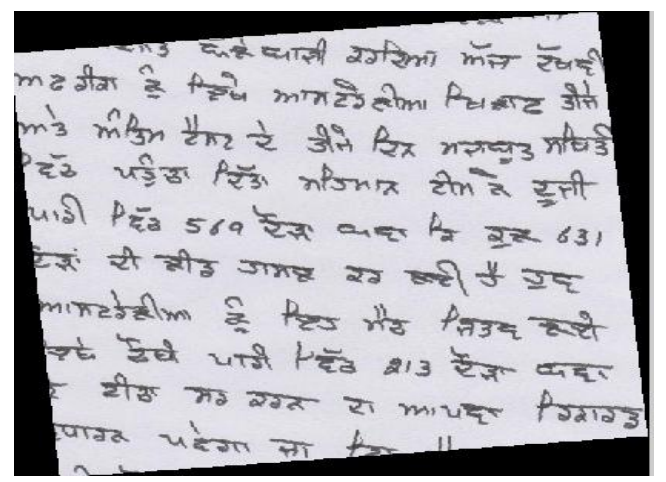

Fig. 1 Skewed Gurmukhi hanwritten text document 


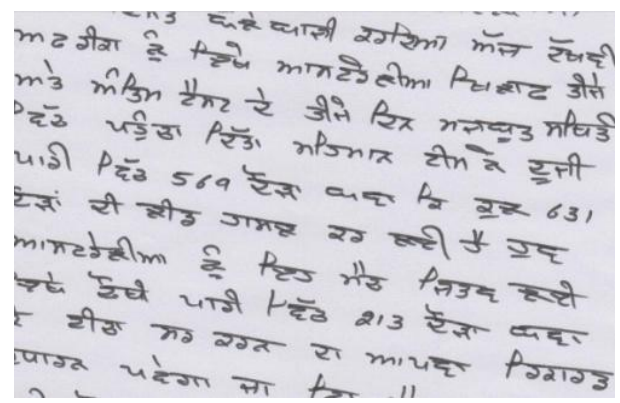

Fig. 2 corrected image of Fig.1

Fig. 1 represents handwritten text. Every line has its own skew angle. Correction of such kind of document is very difficult. The proposed method calculate skew angle of every line and rotate the image according to average value of skew angle

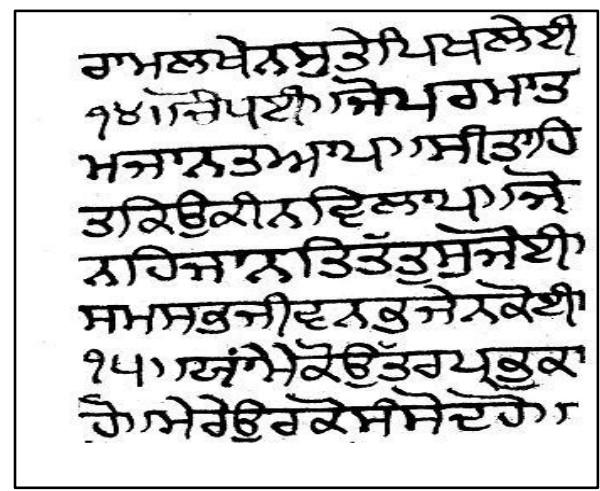

Fig. 3 Skewed Gurmukhi hanwritten text document[9]

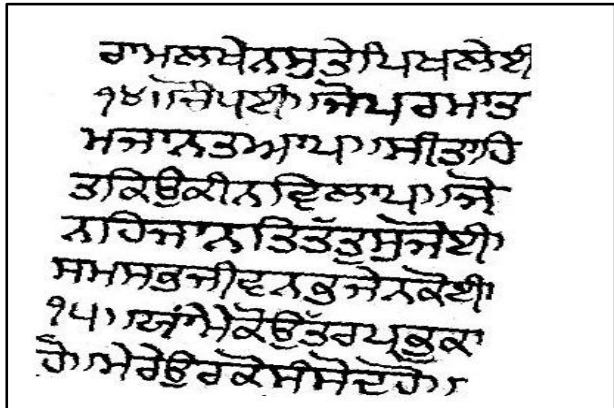

Fig. 4 corrected imageof fig 3 .

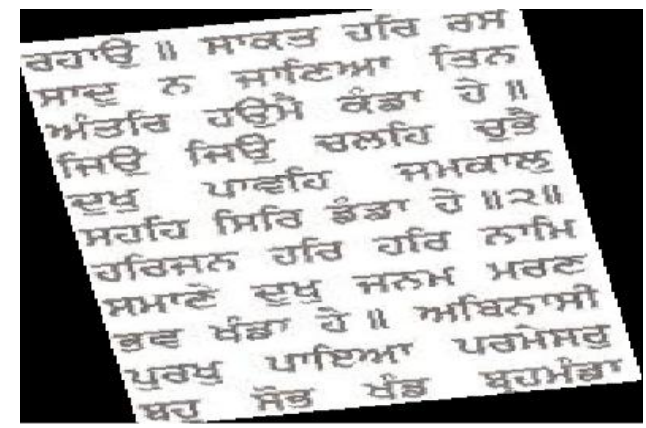

Fig. 5 Skewed Gurmukhi hanwritten text document

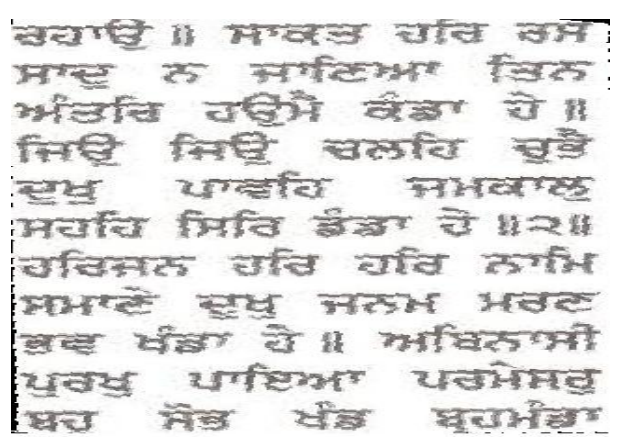

Fig. 6 corrected image of Fig 5

Results of proposed method for handwritten and printed text are given in below table

Table1: Results of proposed method

\begin{tabular}{|l|l|l|l|}
\hline $\begin{array}{l}\text { Actual } \\
\text { Skew } \\
\text { Angle }\end{array}$ & $\begin{array}{l}\text { Detected } \\
\text { Skew } \\
\text { Angle }\end{array}$ & Orientation & Performance \\
\hline 08 & 7.8750 & -ve & $98.4 \%$ \\
\hline 15 & 16.12 & -ve & $100 \%$ \\
\hline 5 & 4.875 & +ve & $97.5 \%$ \\
\hline 10 & 9.87 & -ve & $98.7 \%$ \\
\hline 5.5 & 5.625 & -ve & $100 \%$ \\
\hline 10 & 10.0 & +ve & $100 \%$ \\
\hline 6 & 6 & -ve & $100 \%$ \\
\hline
\end{tabular}

\section{CONCLUSION}

The proposed method provides an efficient, novel and accurate methodology to detect and remove Global skew and local skew. This technique is capable to find the skew angle of individual text line.The main advantage of proposed method is that it works on handwritten document as well as on printed document .It also gives the orientation of skewed lines. The proposed method can calculate the skew angle between 0-20 degrees with $99 \%$ accuracy. The proposed technique can be used to detect Multiskew. However, the proposed method fails for document images containing text with picture.

\section{REFERENCES}

[1] Srihari S. N. and Govindraju V., "Analysis of textual images usingHough Transform", Machine vision Applications 2, 1989, pp 141-153.

[2] T. Akiyama, N. Hagita: "Automated entry systems for printed documents.: Pattern Recognition, 23, 11:11411153,1990

[3] H.S. Baird "The skew angle of printed documents" In: Proc. Soc. Photogr. Sci. Eng., 40:21-24, Rochester, N.Y., USA, 1987

[4] Le D S, Thoma G R and Wechsler H, "Automatic page orientation andskew angle detection for binary document images.” Patter Recognition27, 1994, pp. 1325 - 1344. 
[5] A. Hashizume, P. S. Yeh, and A. Rosenfeld, "A method of detecting the orientation of aligned components," Pattern Recognition Letters, Vol. 4, pp.125-132, 1986.

[6] [6] L. O'Gorman, "The document spectrum for page layout analysis," IEEE Transactions on Pattern Analysis and Machine Intelligence, Vol.15, No.11, pp.1162-1173, 1993.

[7] N. Liolios, N. Fakotkis and G. Kokkinakis, "Improved document skew detetion based on text line connected component clustering," Proc. of Int'l Conf. on Image Processing, Thessaloniki, vol.1, pp.1098-1101, 2001

[8] Pal U, Chaudhuri BB (1996) An improved document skew angle estimation technique. Pattern Recogn Lett 17(8):899-904. doi:10.1016/0167-8655(96)00042-6

[9] Loveleen Kaur,Mandev singh"Comparison of Skew Detection and CorrectionTechniques By applying on Gurmukhi Script" International Journal of Engineering Research \& Technology (IJERT),Vol. 1 Issue 5, ISSN: 2278-0181 . July-2012

[10] Atallah Mahmoud Al-Shatnawi and Khairuddin Omar, "Skew Detection and Correction Technique for Arabic Document Images Based on Centre of Gravity" published in Journal of Computer Science (5):363-368, 2009

[11] Manjunath VN, Kumar GH, Shivakumara" Skew detection technique for binary document images based on Hough transform". International Journal Technologies 13(3):194-200., 2006

[12] Das A. and B. Chanda, "A fast algorithm for skew detection of document images using morphology", International Journal on Document Analysis and Recognition (IJDAR), Vol. 4, 2001, pp 109-114.
[13] Chen S. and R. M. Haralick, "An automatic algorithm for text skew estimation in document images using recursive morphological transforms", In ICIP-94, Austin,Nov., 1994, 139-143.

[14] Prakash K Aithal, Rajesh G, U Dinesh Acharya and Siddalingaswamy P. C," A Fast and Novel Skew Estimation Approach using Radon Transform" International [15Journal of Computer Information Systems and Industrial Management Applications, ISSN 2150-7988 Volume 5 pp. 337-344, 2013

[15] Rajib Ghosh, Gouranga Mandal ," A Novel Approach of Skew Correction for Online Handwritten Words" International Journal of Computer Applications, Volume 48- No.9, pp-0975 - 888 , June 2012

[16] Neha.N ," Language Independent robust skew Detection and CorrectionTechnique for Document Images ",International Journal of Electronics Signals and Systems (IJESS), ISSN: 2231- 5969, Vol-1 Iss-3. 2012

[17] Deepak Kumar, Dalwinder Singh , "Modified Appriach of Hough Transform for skew detection and correction in documented images", International Journal of Research in Computer ScienceISSN 2249-8265 Volume 2 , pp. 3740, 2012

[18] Lehal G S and Madan S "A New Approach to Skew Detection and Correction of Machine Printed Gurmukhi Script", Proc. Int. Conf :, Mumbai, pp. 215$223, .1998$

[19] R. J. Ramteke, Imran Khan Pathan, S. C. Mehrotra ,"Skew Angle Estimation of Urdu DocumentImages: A Moments Based Approach "International Journal of Machine Learning and Computing, Vol.1, No. 1.,2011 